\title{
Acknowledgment to the Reviewers
}

The Editors would like to thank the following referees for the ongoing support in reviewing manuscripts for NeuroImmunoModulation:

K Arimura, Belle Chasse, LA, USA B.G.W. Arnason, Chicago, IL, USA A. Ayalla, Providence, RI, USA

T.M. Badger, Little Rock, AR, USA

\$LH. Barry, Salt Lake City, UT, USA

D. Ben-Nathan, Ness-Ziona, Israel

T. Berg, Oslo, Norway

K. Berzins, Stockholm, Sweden

S. Bhatnager, San Francisco, CA, USA

K.L. Bost, New Orleans, LA, USA

S. Brocke, Bethesda, MD, USA

J.-P. Bourguignon, Liege, Belgium

J.C. Buckingham, London, United Kingdom

D.JJ. Carr, New Orleans, LA, USA D. Chalmers, La Jolla, CA, USA W.G. Clark, Dallas, TX,

USA N. Cohen, Rochester, NY, USA

W.L. Dees, College Station, TX, USA

I. Gantz, Ann Arbor, MI, USA V.K. Ghanta, Birmingham, AL, USA R. Glaser, Berlin,

Germany M. Grazia de Simoni, Milan, Italy 
H. Johnson, Tampa, FL, USA P.E. Jorgensen, Aarhus, Denmark A. Judd, Provo, UT, USA

L. Kapas, Memphis, TN, USA J.L Koenig, Baltimore, MD, USA

J. Licinio, Bethesda, MD, USA K. Lyson, Austin, TX, USA

G.J.M, Maestroni, Locarno, Switzerland M.D. Majewska, Rockville, MD, USA K. Mori, Kyoto, Japan

G.G. Page, Columbus, OH, USA Q.J. Pittman, Calgary, Canada

A.E. Reder, Chicago, IL, USA R.J. Reiter, San Antonio, TX, USA J.N. Richert, Washington, DC, USA R. De Rijle, Bethesda, MD, USA N.R. Rose, Baltimore, MD, USA

E. Spinedi, La Plata, Argentina

A.W. Taylor, Boston, MA, USA 